\title{
A fixed point theorem for a special class of probabilistic contraction
}

\author{
Tatjana Došenović, Aleksandar Takači, Dušan Rakić and Mirjana Brdar
}

\author{
* Correspondence: \\ dosenovic@neobee.net \\ Faculty of Technology, University \\ of Novi Sad, Bulevar cara Lazara 1, \\ Novi Sad, Serbia
}

\author{
Abstract \\ In this paper, a special class of probabilistic contraction will be considered. Using the \\ theory of countable extension of t-norms, we proved a fixed point theorem for such \\ a class of mappings $f: S \rightarrow S$, where $(S, \mathcal{F}, T)$ is a Menger space. \\ Mathematics Subject Classification (2000) \\ $54 \mathrm{H} 25,47 \mathrm{H} 10$
}

Keywords: fixed point, Menger space, $t$-norm, probabilistic $q$-contraction, $q$-contraction $(\varepsilon, \lambda)$-type, strong $b_{n}$-contraction

\section{Introduction}

The notion of a probabilistic metric spaces is introduced in 1942 by K. Menger. The first idea of K. Menger was to use distribution function instead of nonnegative real numbers as values of the metric. Since then, the theory of probabilistic metric spaces has been developed in many directions [1]. The Banach fixed point theorem for contraction mappings has been generalized in different ways, see for example [2-7]. For example, Mihet [4] has worked on the existence and the uniqueness of fixed points of Sehgal contraction, Lj. Cirić at all in [2] worked on a concept of monotone generalized contraction in partially ordered probabilistic metric spaces. Many interesting fixed point results for contraction mappings for singlevalued and multivalued mappings in probabilistic metric spaces can be found in [8].

First, we shall give some definitions and notations.

Definition 1 [9] The ordered pair $(S, \mathcal{F})$ is said to be a probabilistic metric space if $S$ is a nonempty set and $\mathcal{F}: S \times S \rightarrow \Delta^{+}\left(\Delta^{+}\right.$is the set of all distribution functions $F$ such that $F(0)=0$ ) so that the following conditions are satisfied (where $\mathcal{F}(p, q)$ is written by $F_{p, q}$ for every $\left.(p, q) \in S \times S\right)$ :

1. $F_{p, q}(x)=1$ for every $x>0 \Leftrightarrow p=q(p, q \in S)$.

2. $F_{p, q}=F_{q, p}$ for every $p, q \in S$.

3. $F_{p, q}(x)=1$ and $F_{q, r}(y)=1 \Rightarrow F_{p, r}(x+y)=1$ for $p, q, r \in S$ and $x, y \in \mathbb{R}^{+}$.

Definition $2[9]$ A mapping $T:[0,1] \times[0,1] \rightarrow[0,1]$ is called a triangular norm (a t-norm) if the following conditions are satisfied:

$$
T(a, 1)=\text { a for every } a \in[0,1]
$$

(c) 2011 Došenovićć et al; licensee Springer. This is an Open Access article distributed under the terms of the Creative Commons Attribution License (http://creativecommons.org/licenses/by/2.0), which permits unrestricted use, distribution, and reproduction in any medium, provided the original work is properly cited. 
$T(a, b)=T(b, a)$ for every $a, b \in[0,1]$

$a \geq b, c \geq d \Rightarrow T(a, c) \geq T(b, d)(a, b, c, d \in[0,1]) ;$

$T(a, T(b, c))=T(T(a, b), c)(a, b, c \in[0,1])$.

Definition 3 If $T$ is a $t$-norm, then its dual t-conorm $S:[0,1]^{2} \rightarrow[0,1]$ is given by

$$
S(x, y)=1-T(1-x, 1-y) .
$$

Example 1 Basic examples are as follows:

$$
\begin{aligned}
T_{M}(x, y) & =\min (x, y), S_{M}(x, y)=\max (x, y) \\
T_{P}(x, y) & =x \cdot y, S_{P}(x, y)=x+y-x y \\
T_{L}(x, y) & =\max (x+y-1,0) \\
S_{L}(x, y) & =\min (x+y, 1) \\
T_{D}(x, y) & = \begin{cases}\min (x, y) & \text { if } \max (x, y)=1, \\
0 & \text { otherwise }\end{cases} \\
S_{D}(x, y) & = \begin{cases}\max (x, y) & \text { if } \min (x, y)=0, \\
1 & \text { otherwise }\end{cases}
\end{aligned}
$$

Definition 4 [9] A Menger space is an ordered triple $(S, \mathcal{F}, T)$, where $(S, \mathcal{F})$ is a probabilistic metric space, $T$ is a $t$-norm and the generalized triangle inequality

$$
F_{p, q}(x+y) \geq T\left(F_{p, r}(x), F_{q, r}(y)\right)
$$

holds for every $p, q, r \in S$ and every $x>0, y>0$.

Definition 5 (i) $A$ t-norm $T$ is said to be strictly monotone if $T(x, y)<T(x, z)$ whenever $x \in(0,1)$ and $y<z$.

(ii) $A$ t-norm $T$ is called strict if it is continuous and strictly monotone.

(iii) A continuous t-norm $T$ is called Archimedean if $T(x, x)<x$, for all $x \in(0,1)$.

Theorem 1 A function $T:[0,1]^{2} \rightarrow[0,1]$ is a continuous Archimedean t-norm if and only if there exists a continuous, strictly decreasing function $\mathbf{t}:[0,1] \rightarrow[0,+\infty]$ with $\mathbf{t}(1)=0$ such that for all $x, y \in[0,1]$

$$
T(x, y)=\mathbf{t}^{-1}(\min (\mathbf{t}(x)+\mathbf{t}(y), \mathbf{t}(0))) .
$$

The function $\mathbf{t}$ is then called an additive generator of $T$; it is uniquely determined by $T$ up to a positive multiplicative constant.

The $(\varepsilon, \lambda)$-topology in $S$ is introduced by the family of neighborhoods of $v \in S$, $\mathcal{U}_{v}=\left\{U_{v}(\varepsilon, \lambda)\right\}_{(\varepsilon, \lambda) \in \mathbb{R}_{+} \times(0,1)}$, where

$$
U_{v}(\varepsilon, \lambda)=\left\{u ; F_{u, v}(\varepsilon)>1-\lambda\right\} .
$$

If a $t$-norm $T$ is such that $\sup _{x<1} T(x, x)=1$, then $\left\{\mathcal{U}_{v}\right\}_{v \in S}$ defines on $S$ a metrizable topology.

Definition 6 A sequence $\left\{x_{n}\right\}_{n \in \mathbb{N}}$ in $S$ is a Cauchy sequence if and only if for every $\varepsilon$ $>0$ and $\lambda \in(0,1)$ there exists $n_{0}(\varepsilon, \lambda) \in \mathbb{N}$ such that for every $n \geq n_{0}(\varepsilon, \lambda)$ and every $p$ $\in \mathbb{N}$

$$
F_{x_{n+p}, x_{n}}(\varepsilon)>1-\lambda
$$


If a probabilistic metric space $(S, \mathcal{F})$ is such that every Cauchy sequence $\left\{x_{n}\right\}_{n \in \mathbb{N}}$ in $S$ converges in $S$, then $(S, \mathcal{F})$ is a complete space.

In [10], a class of $t$-norms is introduced, which is useful in the fixed point theory in probabilistic metric spaces.

Let $T$ be a $t$-norm and $T_{n}:[0,1] \rightarrow[0,1](n \in \mathbb{N})$ be defined in the following way:

$$
T_{1}(x)=T(x, x), \quad T_{n+1}(x)=T\left(T_{n}(x), x\right) \quad(n \in \mathbb{N}, x \in[0,1]) .
$$

We say that $t$-norm $T$ is of $H$-type if the family $\left\{T_{n}(x)\right\}_{n \in \mathbb{N}}$ is equicontinuous at $x=$ 1.

One of the most important results for the fixed point theory in metric space $(M, d)$ is the Banach contraction principle.

A mapping $f: M \rightarrow M$ is said to be a $q$-contraction if there exists $q \in[0,1)$ such that

$$
d(f x, f y) \leq q d(x, y)
$$

for every $x, y \in M$.

Every $q$-contraction $f: M \rightarrow M$ on a complete metric space $(M, d)$ has one and only one fixed point.

Sehgal and Bharucha-Reid introduced in [11] the notion of a probabilistic $q$-contraction $(q \in(0,1))$ in probabilistic metric space.

Definition 7 Let $(S, \mathcal{F})$ be a probabilistic metric space. A mapping $f: S \rightarrow S$ is a probabilistic q-contraction if

$$
F_{f p_{1}, f p_{2}}(x) \geq F_{p_{1}, p_{2}}\left(\frac{x}{q}\right)
$$

for every $p_{1}, p_{2} \in S$ and every $x \in \mathbb{R}$.

The first fixed point theorem in probabilistic metric space was proved by Sehgal and Bharucha-Reid in [11].

Theorem 2 Let $\left(S, \mathcal{F}, T_{M}\right)$ be a complete Menger space and $f: S \rightarrow S$ a probabilistic $q$-contraction. Then, there exists a unique fixed point $x$ of the mapping $f$ and $x=\lim _{n \rightarrow \infty} f^{n} p$ for every $p \in S$.

In [12], Mihet introduced the following definition.

Definition 8 Let $(S, \mathcal{F})$ be a probabilistic metric space. A mapping $f: S \rightarrow S$ is said to be a q-contraction of $(\varepsilon, \lambda)$-type, if the following implication holds for every $p_{1}, p_{2} \in$ $S:$

$$
(\forall \varepsilon>0)(\forall \lambda \in(0,1))\left(F_{p_{1}, p_{2}}(\varepsilon)>1-\lambda \Rightarrow F_{f p_{1}, f p_{2}}(q \varepsilon)>1-q \lambda\right) .
$$

It is obvious that (1) implies that $f$ is a probabilistic $q$-contraction.

In [12], the following theorem was proved.

Theorem 3 Let $\left(S, \mathcal{F}, T_{L}\right)$ be a complete Menger space and $f: S \rightarrow S$ a q-contraction of $(\varepsilon, \lambda)$-type. Then, there exists a unique fixed point $x \in S$ of the mapping $f$ and $x=\lim _{n \rightarrow \infty} f^{n} p$ for every $p \in S$.

In [8], Hadžić and Pap proved that Mihet's theorem holds for a more general class of Menger space. In order to formulate the theorem, the notion of geometrical convergence of a $t$-norm is defined. 
Definition 9 We say that a $t$-norm $T$ is geometrically convergent if

$$
\lim _{n \rightarrow \infty} \mathbf{T}_{i=n}^{\infty}\left(1-q^{i}\right)=1
$$

for every $q \in(0,1)$.

Theorem 4 Let $(S, \mathcal{F}, T)$ be a complete Menger space such that and $f: S \rightarrow S$ a qcontraction of $(\varepsilon, \lambda)$-type. If $T$ is geometrically convergent, then there exists a unique fixed point $x \in S$ of the mapping $f$ and $x=\lim _{n \rightarrow \infty} f^{n} p$ for every $p \in S$.

In this paper, the definition of a strong $\left(b_{n}\right)$-contraction is introduced. Using the theory of countable extension of a $t$-norm given in [8], we prove a fixed point theorem where the mapping $f: S \rightarrow S$ is a strong $\left(b_{n}\right)$-contraction, and $(S, \mathcal{F}, T)$ is a complete Menger space and $T$ satisfies an additional condition. In corollaries, we prove that this condition is satisfied if $T$ belongs to the class of $t$-norms of Dombi, Aczél-Alsina and Sugeno-Weber.

\section{Countable extension of $\boldsymbol{t}$-norms}

Each $t$-norm $T$ can be extended (by associativity see [13]) in a unique way to an nary operation taking for $\left(x_{1}, \ldots, x_{n}\right) \in 0[1]^{n}$ the values

$$
\mathbf{T}_{i=1}^{0} x_{i}=1, \mathbf{T}_{i=1}^{n} x_{i}=T\left(\mathbf{T}_{i=1}^{n-1} x_{i}, x_{n}\right) .
$$

A $t$-norm $T$ can be extended to a countable infinitary operation taking for any sequence $\left(x_{n}\right)_{n \in \mathbb{N}}$ from $[0,1]$ the value

$$
\mathbf{T}_{i=1}^{\infty} x_{i}=\lim _{n \rightarrow \infty} \mathbf{T}_{i=1}^{n} x_{i} .
$$

The sequence $\left(\mathrm{T}_{i=1}^{n} x_{i}\right)_{n \in \mathbb{N}}$ is nonincreasing and bounded from below, and hence, the limit $\mathbf{T}_{i=1}^{\infty} x_{i}$ exists.

In the fixed point theory (see [8]), it is of interest to investigate the classes of $t$ norms $T$ and sequences $\left(x_{n}\right)$ from the interval $[0,1]$ such that $\lim _{n \rightarrow \infty} x_{n}=1$ and

$$
\lim _{n \rightarrow \infty} \mathbf{T}_{i=n}^{\infty} x_{i}=\lim _{n \rightarrow \infty} \mathbf{T}_{i=1}^{\infty} x_{n+i}=1 .
$$

It is obvious that

$$
\lim _{n \rightarrow \infty} \mathbf{T}_{i=n}^{\infty} x_{i}=1 \Leftrightarrow \sum_{i=1}^{\infty}\left(1-x_{i}\right)<\infty
$$

for $T=T_{L}$ and $T=T_{p}$.

For $T \geq T_{L}$, we have the following implication

$$
\lim _{n \rightarrow \infty} \mathbf{T}_{i=n}^{\infty} x_{i}=1 \Rightarrow \sum_{i=1}^{\infty}\left(1-x_{i}\right)<\infty .
$$

Important classes of $t$-norms are given in the following example. 
Example 2 (i) The Dombi family of t-norms $\left(T_{\lambda}^{D}\right)_{\lambda \in[0, \infty]}$ is defined by

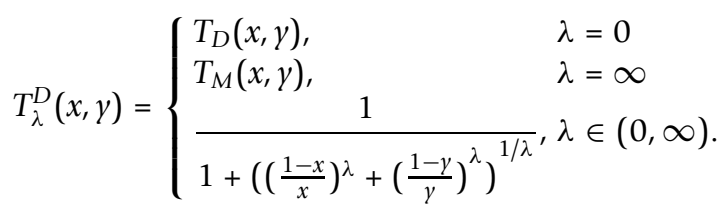

(ii) The Aczél-Alsina family of t-norms $\left(T_{\lambda}^{A A}\right)_{\lambda \in[0, \infty]}$ is defined by

$$
T_{\lambda}^{A A}(x, y)= \begin{cases}T_{D}(x, y), & \lambda=0 \\ T_{M}(x, y), & \lambda=\infty \\ e^{-\left((-\log x)^{\lambda}+(-\log y)^{\lambda}\right)^{1 / \lambda}}, & \lambda \in(0, \infty) .\end{cases}
$$

(iii) The family $\left(T_{\lambda}^{S W}\right)_{\lambda \in[-1, \infty]}$ of Sugeno-Weber -norms is given by

$$
T_{\lambda}^{S W}(x, y)= \begin{cases}T_{D}(x, y), & \lambda=-1 \\ T_{P}(x, y), & \lambda=\infty \\ \max \left(0, \frac{x+y-1+\lambda x y}{1+\lambda}\right), & \lambda \in(-1, \infty) .\end{cases}
$$

(iv) The Schweizer-Sklar family of t-norms $\left(T_{\lambda}^{S S}\right)_{\lambda \in[-\infty, \infty]}$ is defined by

$$
T_{\lambda}^{S S}(x, y)= \begin{cases}T_{M}(x, y), & \lambda=-\infty \\ T_{P}(x, y), & \lambda=0 \\ \left(\max \left(x^{\lambda}+y^{\lambda}-1,0\right)\right)^{1 / \lambda}, & \lambda \in(-\infty, 0) \cup(0, \infty) \\ T_{D}(x, y), & \lambda=\infty .\end{cases}
$$

The condition $T \geq T_{L}$ is fulfilled by the families $\left(T_{\lambda}^{S S}\right)_{\lambda \in(-\infty, 1)},\left(T_{\lambda}^{S W}\right)_{\lambda \in[0, \infty]}$.

On the other side, there exists a member of the family $\left(T_{\lambda}^{D}\right)_{\lambda \in(0, \infty)}$ which is incomparable with $T_{L}$, and there exists a member of the family $\left(T_{\lambda}^{A A}\right)_{\lambda \in(0, \infty)}$ which is incomparable with $T_{L}$.

In [8], the following results are obtained:

(a) If $\left(T_{\lambda}^{D}\right)_{\lambda \in(0, \infty)}$ is the Dombi family of $t$-norms and $\left(x_{n}\right)_{n \in \mathbb{N}}$ a sequence of elements from $(0,1]$ such that $\lim _{n \rightarrow \infty} x_{n}=1$, then we have the following equivalence

$$
\sum_{n=1}^{\infty}\left(1-x_{n}\right)^{\lambda}<\infty \Leftrightarrow \lim _{n \rightarrow \infty}\left(T_{\lambda}^{D}\right)_{i=n}^{\infty} x_{i}=1 .
$$

(b) If $\left(T_{\lambda}^{S W}\right)_{\lambda \in(-1, \infty]}$ is the Sugeno-Weber family of $t$-norms and $\left(x_{n}\right)_{n \in \mathbb{N}}$ a sequence of elements from $(0,1]$ such that $\lim _{n \rightarrow \infty} x_{n}=1$, then we have the following equivalence

$$
\sum_{n=1}^{\infty}\left(1-x_{n}\right)<\infty \Leftrightarrow \lim _{n \rightarrow \infty}\left(T_{\lambda}^{S W}\right)_{i=n}^{\infty} x_{i}=1
$$

(c) The equivalence (3) holds also for the family $\left(T_{\lambda}^{A A}\right)_{\lambda \in(0, \infty)}$, i.e.,

$$
\sum_{n=1}^{\infty}\left(1-x_{n}\right)^{\lambda}<\infty \Leftrightarrow \lim _{n \rightarrow \infty}\left(T_{\lambda}^{A A}\right)_{i=n}^{\infty} x_{i}=1 .
$$

In [8], the following Proposition is obtained. 
Proposition 1 Let $\left(x_{n}\right)_{n \in \mathbb{N}}$ be a sequence of numbers from $[0,1]$ such that $\lim _{n \rightarrow \infty} x_{n}=1$ and t-norm $T$ is of H-type. Then, $\lim _{n \rightarrow \infty} \mathbf{T}_{i=n}^{\infty} x_{i}=\lim _{n \rightarrow \infty} \mathbf{T}_{i=1}^{\infty} x_{n+i}=1$.

In the fixed point theory in probabilistic spaces, it is of interest to investigate condition (2) for a special sequence $\left(1-q^{n}\right)_{n \in \mathbb{N}}$ for $q \in(0,1)$.

In [14], the following Proposition is proved.

Proposition 2 If for a $t$-norm $T$ there exists $q_{0} \in(0,1)$ such that

$$
\lim _{n \rightarrow \infty} \mathbf{T}_{i=n}^{\infty}\left(1-q_{0}^{i}\right)=1,
$$

then

$$
\lim _{n \rightarrow \infty} \mathbf{T}_{i=n}^{\infty}\left(1-q^{i}\right)=1
$$

for every $q \in(0,1)$.

In [8] the following definition is given.

Since $\lim _{n \rightarrow \infty}\left(1-q^{n}\right)=1$ and $\sum_{n=1}^{\infty}\left(1-\left(1-q^{n}\right)\right)<\infty$ for every $s>0$ it follows that all $t$-norms from the family

$$
\mathcal{T}_{0}=\bigcup_{\lambda \in(0, \infty)}\left\{T_{\lambda}^{D}\right\} \bigcup \bigcup_{\lambda \in(0, \infty)}\left\{T_{\lambda}^{A A}\right\} \bigcup \mathcal{T}^{H} \bigcup_{\lambda \in(-1, \infty]}\left\{T_{\lambda}^{S W}\right\}
$$

are geometrically convergent, where $\mathcal{T}^{H}$ is the class of all $t$-norms of $H$-type.

\section{A fixed point theorem}

Definition 10 Let $(S, \mathcal{F})$ be a probabilistic metric space and $\left(b_{n}\right)_{n \in \mathbb{N}}$ a sequence from $(0,1)$ such that $\lim _{n \rightarrow \infty} b_{n}=1$. The mapping $f: S \rightarrow S$ is a strong $\left(b_{n}\right)$-contraction if the following implication holds

$$
\begin{gathered}
(\exists q \in(0,1))(\forall n \in \mathbb{N})(\forall \varepsilon>0)(\forall x, y \in S) \\
F_{x, y}(\varepsilon)>b_{n} \Rightarrow F_{f x, f y}(q \varepsilon)>b_{n+1} .
\end{gathered}
$$

Each $q$-contraction $(\varepsilon, \lambda)$-type is a strong $b_{n}$-contraction, where $q \in(0,1)$, if a sequence $\left(b_{n}\right)_{n \in \mathbb{N}}$ is defined in the following way:

$$
b_{m+1}=1-q^{m} \lambda, \quad m \in \mathbb{N}, \quad \text { for some } \lambda \in(0,1) .
$$

Theorem 5 Let $(S, \mathcal{F}, T)$ be a complete Menger space and $\left(b_{n}\right)_{n \in \mathbb{N}}$ a sequence from $(0,1)$ such that $\lim _{n \rightarrow \infty} b_{n}=1$. If $t$-norm $T$ satisfies the following condition

$$
\lim _{n \rightarrow \infty} \mathbf{T}_{i=n}^{\infty} b_{i}=1
$$

and $f: S \rightarrow S$ is a strong $\left(b_{n}\right)$-contraction, then there exists a unique fixed point $x \in S$ of the mapping $f$ and $x=\lim _{n \rightarrow \infty} f^{n} p$ for every $p \in S$.

Proof: We will show that mapping $f: S \rightarrow S$ is uniformly continuous since $f$ is a strong $\left(b_{n}\right)$-contraction. Let $\delta>0$ and $\lambda \in(0,1)$ be given. Since $\lim _{n \rightarrow \infty} b_{n}=1$, there exists $m \in \mathbb{N}$ such that $b_{m+1}>1-\lambda$. Let $\varepsilon=\frac{\delta}{q}, q \in(0,1)$. 
Then, the following implication holds

$$
F_{x, y}(\varepsilon)>b_{m} \Rightarrow F_{f x, f y}(q \varepsilon)=F_{f x, f y}(\delta)>b_{m+1}>1-\lambda .
$$

Let $N(\varepsilon, \lambda)=\left\{(u, v): u, v \in S, F_{u, v}(\varepsilon)>1-\lambda\right\}$.

It follows that $(x, y) \in N\left(\frac{\delta}{q}, 1-b_{m}\right) \Rightarrow(f x, f y) \in N(\delta, \lambda)$, which means that a mapping $f$ is uniformly continuous.

We shall prove that a sequence $\left(f^{n} p\right)_{n \in \mathbb{N}}$ is Cauchy sequence for every $p \in S$, i.e., that for every $\varepsilon>0$ and $\lambda \in(0,1)$, there exists $n_{0}(\varepsilon, \lambda) \in \mathbb{N}$ such that for every $n>n_{0}(\varepsilon, \lambda)$ and $r \in \mathbb{N}$ the following condition is satisfied

$$
F_{f^{n} p, f^{n+r} p}(\varepsilon)>1-\lambda \text {. }
$$

Since $t$-norm $T$ satisfies the condition $\lim _{n \rightarrow \infty} \mathbf{T}_{i=n}^{\infty} b_{i}=1$, it follows that there exists $m_{0}$ $\in \mathbb{N}$ such that

$$
\mathbf{T}_{i=m_{0}}^{\infty} b_{i}>1-\lambda
$$

Let $p \in S$. Since $F_{p, f p} \in \Delta^{+}$, there exists $\eta$ such that

$$
F_{p, f p}(\eta)>b_{m_{0}} .
$$

The mapping $f$ is a strong $\left(b_{n}\right)$-contraction and (8) implies

$$
F_{f p, f^{2} p}(q \eta)>b_{m_{0}+1} \text {. }
$$

Continuing in this way, we got that for every $k \in \mathbb{N}$

$$
F_{f^{k}, f^{k+1} p}\left(q^{k} \eta\right)>b_{m_{0}+k}
$$

Let $k_{0} \in \mathbb{N}$ be such that $\sum_{k=k_{0}}^{\infty} q^{k}<\frac{\varepsilon}{\eta}$ and $k_{0}>m_{0}$. Then for every $l \in \mathbb{N}$ and every $r$ $\geq 2$, it follows

$$
\begin{aligned}
F_{f^{k_{0}+l} p, f^{k_{0}+l+r} p}(\varepsilon) & \geq F_{f^{k_{0}+l} p, f^{k_{0}+l+r} p}\left(\eta \sum_{k=k_{0}}^{\infty} q^{k}\right) \\
& \geq F_{f^{k_{0}+l} p, f^{k_{0}+l+r} p}\left(\eta \sum_{k=k_{0}+l}^{k_{0}+l+r-1} q^{k}\right) \\
& \geq \underbrace{T(T \ldots T}_{(r-1)-t i m e s}\left(F_{f^{k_{0}+l} p, f^{k_{0}+l+1} p}\left(\eta q^{k_{0}+l}\right),\right. \\
& \left.\ldots F_{f^{k_{0}+l+1} p, f^{k_{0}+l+2} p}\left(\eta q^{k_{0}+l+1}\right)\right) \\
& \left.\geq F_{f^{k_{0}+l+r-1} p, f^{k_{0}+l+r} p}\left(\eta q^{k_{0}+l+r-1}\right)\right) \\
& \mathbf{T}_{i=m_{0}}^{\infty} b_{i} \\
& 1-\lambda .
\end{aligned}
$$

This means that the sequence $\left(f^{n} p\right)_{n \in \mathbb{N}}$ is a Cauchy, and since the space is complete, there exists $x \in S$ such that $x=\lim _{n \rightarrow \infty} f^{n} p$. From the continuity of the mapping $f$, it follows that $x=f x$. Let $y=f y$, for $y \in S$. It remains to be proved that $x=y$, i.e., we have to prove that $F_{x, y}(\varepsilon)>1-\lambda$ for every $\varepsilon>0$ and every $\lambda \in(0,1)$. Let $\varepsilon>0$ and $\lambda \in(0$, 
1) be given. From the condition $\lim _{n \rightarrow \infty} b_{n}=1$, it follows that there exists $m \in \mathbb{N}$ such that $b_{m}>1-\lambda$. Since $F_{x, y} \in \Delta^{+}$, there exists $\delta>0$ such that $F_{x, y}(\delta)>b_{m}$. Because $q \in$ $(0,1)$, there exists $k_{0}$ such that $\varepsilon>q^{k} \delta$, for every $k \geq k_{0}$ and there exists $k_{1}>k_{0}$ such that $b_{m+k_{1}}>b_{m}$. From the Definition 10, it follows $F_{x, y}(\varepsilon)>F_{x, y}\left(q^{k_{1}} \delta\right)>b_{m+k_{1}}>b_{m}>1-\lambda$.

The following Corollary is in fact Theorem 4.

Corollary 1 Let $(S, \mathcal{F}, T)$ be a complete Menger space and $f: S \rightarrow S$ is a q-contraction $(\varepsilon, \lambda)$-type. If $t$-norm $T$ is geometrically convergent, then there exists a unique fixed point $x \in S$ of the mapping $f$ and $x=\lim _{n \rightarrow \infty} f^{n} p$ for every $p \in S$.

Proof: Let $\left(b_{n}\right)_{n \in \mathbb{N}}$ be a sequence defined in the following way

$$
b_{m+1}=1-q^{m} \lambda,
$$

$m \in \mathbb{N}$, for some $\lambda \in(0,1)$. Since $\lambda \in(0,1)$, it follows that $q^{i} \lambda<q^{i}$ and so

$$
\mathbf{T}_{i=1}^{\infty}\left(1-q^{i} \lambda\right)>\mathbf{T}_{i=1}^{\infty}\left(1-q^{i}\right) .
$$

From that it follows that $\lim _{n \rightarrow \infty} \mathbf{T}_{i=n}^{\infty}\left(1-q^{i} \lambda\right)=\lim _{n \rightarrow \infty} \mathbf{T}_{i=n}^{\infty} b_{i}=1$, and the whole conditions of previous theorem are satisfied.

Remark In [12], Mihet introduced the following definition.

Definition 11 Let $(S, \mathcal{F})$ be a probabilistic space and $\left(b_{n}\right)_{n \in \mathbb{N}}$ a sequence in $(0,1)$ such that $b_{n}>1$. We say that the mapping $f: S \rightarrow S$ is a $\left(b_{n}\right)$-contraction if for all $n \in$ $\mathbb{N}$, there exists $k_{n} \in(0,1)$ such that for every $p, q \in S$ and every $t>0$

$$
F_{p, q}(t)>b_{n} \Rightarrow F_{f p, f q}\left(k_{n} t\right)>b_{n} .
$$

In [12], Mihet proved that a $b_{n}$-contraction in a complete Menger space under a $t$ norm $T=T_{H}$ has a unique fixed point.

The class of strong $\left(b_{n}\right)$-contraction is strictly included in the class of $\left(b_{n}\right)$-contraction (for more details see [15]). For future work, it would be interesting to combine Theorem 5 with the result given above, i.e., to use $\left(b_{n}\right)$-contraction as the mapping and a more general class of $t$-norms (for example, the one used in Theorem 5 ).

Corollary 2 Let $\left(S, \mathcal{F},\left(T_{\lambda}^{D}\right)_{\lambda \in(0, \infty)}\right)$ be a complete Menger space and $\left(b_{n}\right)_{n \in \mathbb{N}} a$ sequence from $(0,1)$ such that $\lim _{n \rightarrow \infty} b_{n}=1$. If $\sum_{i=1}^{\infty}\left(1-b_{i}\right)^{\lambda}<\infty$ and $f: S \rightarrow S$ is a strong $\left(b_{n}\right)$-contraction, then there exists a unique fixed point $x \in S$ of the mapping $f$ and $x=\lim _{n \rightarrow \infty} f^{n} p$ for every $p \in S$.

Proof: From equivalence (3), we have

$$
\sum_{i=1}^{\infty}\left(1-b_{i}\right)^{\lambda}<\infty \Leftrightarrow \lim _{n \rightarrow \infty}\left(T_{\lambda}^{D}\right)_{i=n}^{\infty} b_{i}=1
$$

Since $\sum_{i=1}^{\infty}\left(1-b_{i}\right)^{\lambda}<\infty$ is satisfied, all the conditions of previous theorem are fulfilled.

Corollary 3 Let $\left(S, \mathcal{F},\left(T_{\lambda}^{A A}\right)_{\lambda \in(0, \infty)}\right)$ be a complete Menger space and $\left(b_{n}\right)_{n \in \mathbb{N}} a$ sequence from $(0,1)$ such that $\lim _{n \rightarrow \infty} b_{n}=1$. If $\sum_{i=1}^{\infty}\left(1-b_{i}\right)^{\lambda}<\infty$ and $f: S \rightarrow S$ is a 
strong $\left(b_{n}\right)$-contraction, then there exists a unique fixed point $x \in S$ of the mapping $f$ and $x=\lim _{n \rightarrow \infty} f^{n} p$ for every $p \in S$.

Proof: From equivalence (5), we have

$$
\sum_{i=1}^{\infty}\left(1-b_{i}\right)^{\lambda}<\infty \Leftrightarrow \lim _{n \rightarrow \infty}\left(T_{\lambda}^{A A}\right)_{i=n}^{\infty} b_{i}=1
$$

Since $\sum_{i=1}^{\infty}\left(1-b_{i}\right)^{\lambda}<\infty$ is satisfied all the conditions of previous theorem are fulfilled.

Corollary 4 Let $\left(S, \mathcal{F},\left(T_{\lambda}^{S W}\right)_{\lambda \in(-1, \infty]}\right)$ be a complete Menger space and $\left(b_{n}\right)_{n \in \mathbb{N}} a$ sequence from $(0,1)$ such that $\lim _{n \rightarrow \infty} b_{n}=1$. If $\sum_{i=1}^{\infty}\left(1-b_{i}\right)<\infty$ and $f: S \rightarrow S$ is a strong $\left(b_{n}\right)$-contraction, then there exists a unique fixed point $x \in S$ of the mapping $f$ and $x=\lim _{n \rightarrow \infty} f^{n} p$ for every $p \in S$.

Proof: From equivalence (4), we have

$$
\sum_{i=1}^{\infty}\left(1-b_{i}\right)<\infty \Leftrightarrow \lim _{n \rightarrow \infty}\left(T_{\lambda}^{S W}\right)_{i=n}^{\infty} b_{i}=1
$$

Since $\sum_{i=1}^{\infty}\left(1-b_{i}\right)<\infty$ is satisfied, all the conditions of previous theorem are fulfilled.

Corollary 5 Let $(S, \mathcal{F}, T)$ be a complete Menger space and $\left(b_{n}\right)_{n \in \mathbb{N}}$ a sequence from $(0,1)$ such that $\lim _{n \rightarrow \infty} b_{n}=1$. If t-norm $T$ is of H-type and $f: S \rightarrow S$ is a strong $\left(b_{n}\right)$-contraction, then there exists a unique fixed point $x \in S$ of the mapping $f$ and $x=\lim _{n \rightarrow \infty} f^{n} p$ for every $p \in S$.

Proof: The proof results from the Proposition 1 directly.

\section{Probabilistic metric space related to decomposable measure}

Let $\mathcal{A}$ be a $\sigma$-algebra of subsets of a given set $\Omega$. A classical measure is a set function $m: \mathcal{A} \rightarrow[0, \infty]$ such that $m(\varnothing)=0$ and

$$
m\left(\bigcup_{i=1}^{\infty} A_{i}\right)=\sum_{i=1}^{\infty} m\left(A_{i}\right)
$$

for every sequence $\left(A_{i}\right)_{i \in \mathbb{N}}$ of pairwise disjoint set from $\mathcal{A}$.

Definition 12 Let $S$ be a t-conorm. A $\mathcal{S}$-decomposable measure $m$ is a set function $m: \mathcal{A} \rightarrow[0,1]$ such that $m(\varnothing)=0$ and

$$
m(A \cup B)=\mathcal{S}(m(A), m(B))
$$

for every $A, B \in \mathcal{A}$ and $A \cap B=\varnothing$.

A measure $m$ is of (NSA)-type if and only if $s \circ m$ is a finite additive measure, where $\mathbf{s}$ is an additive generator of the t-conorm $S$, which is continuous, nonstrict, and Archimedean and with respect to which $m$ is decomposable $(\mathbf{s}(1)=1)$.

Proposition 3 Let $(\Omega, \mathcal{A}, m)$ be a measure space, where $m$ is a continuous $\mathcal{A}$ decomposable measure of (NSA)-type with monotone increasing generator $\mathbf{s}$. Then, $(S, \mathcal{F}, T)$ is a Menger space, where $\mathcal{F}$ and $t$-norm $T$ are given in the following way 


$$
\begin{gathered}
F_{\hat{X}, \hat{Y}}(u)=m\{\omega: \omega \in \Omega, d(X(\omega), Y(\omega))<u\} \\
=m\{d(X, Y)<u\} \\
F_{\hat{X}, \hat{Y}}(u)=m\{\omega: \omega \in \Omega, d(X(\omega), Y(\omega))<u\} \\
=m\{d(X, Y)<u\}
\end{gathered}
$$

(for every $\hat{X}, \hat{Y} \in S, u \in \mathbb{R}$ ),

$$
T(x, y)=\mathbf{s}^{-1}(\max (0, \mathbf{s}(x)+\mathbf{s}(y)-1))
$$

for every $x, y \in[0,1]$.

Proposition 4 Let $(\Omega, \mathcal{A}, m)$ be as in Proposition 3 and $(M, d)$ be a complete separable metric space. Then, $(S, \mathcal{F}, T)$ from Proposition 3 is a complete probabilistic metric space.

Example 3 Let $(\Omega, \mathcal{A}, P)$ be a probability measure space, $(M, d)$ a separable metric space, and $\mathcal{B}_{M}$ the family of Borel subsets of $M$. A mapping $f: \Omega \times M \rightarrow M$ is a random operator if for every $C \in \mathcal{B}_{M}$ and every $x \in M$

$$
\{\omega ; \omega \in \Omega, f(\omega, x) \in C\} \in \mathcal{A},
$$

i.e., if the mapping $\omega \mapsto f(\omega, x)$ is measurable on $\Omega$. A random operator $f: \Omega \times M \rightarrow$ $M$ is continuous if for every $\omega \in \Omega$ the mapping $x \mapsto f(\omega, x)$ is continuous on $M$.

If $f: \Omega \times M \rightarrow M$ is a continuous random operator, then for every measurable mapping $X: \Omega \rightarrow M$ the mapping $\omega \mapsto f(\omega, X(\omega))$ is measurable on $\Omega$.

Let $S$ be the set of all equivalence classes of measurable mappings $X: \Omega \rightarrow M$ and let $f$ be a continuous random operator. The mapping $\widehat{f}: S \rightarrow S$, defined by

$$
\widehat{(f X})(\omega)=f(\omega, X(\omega)) \text { for every } \widehat{X} \in S(\omega \in \Omega, X \in \widehat{X}),
$$

is the so-called Nemytskij operator of $f$. If $f: \Omega \times M \rightarrow M$ is a random operator, then a measurable mapping $X: \Omega \rightarrow M$ is a random fixed point of the mapping $f$ if

$$
X(\omega)=f(\omega, X(\omega)) \text { a.e. }
$$

If $f$ is a continuous random operator, then (12) holds if and only if $\widehat{X}=\widehat{f} \widehat{X}, X \in \widehat{X}$. In this case, the problem of the existence of a random fixed point of a continuous random operator $f$ reduces to the problem of the existence of a fixed point of the Nemytskij operator $\widehat{f}$ of $f$.

Corollary 6 Let $(\Omega, \mathcal{A}, m)$ be a measure space, where $m$ is a continuous $\mathcal{S}$ decomposable measure of (NSA)-type, $\mathbf{s}$ is a monotone increasing additive generator of $\mathcal{S},(M, d)$ a complete separable metric space and $f: \Omega \times S \rightarrow M$ a random operator such that for some $q \in(0,1)$ and every measurable mappings $X, Y: \Omega \rightarrow M$

$$
\begin{gathered}
(\forall u>0)(\forall n \in \mathbb{N})(m\{\omega ; \omega \in \Omega, d(X(\omega), Y(\omega))<u\})>b_{n} \\
\Rightarrow m(\{\omega ; \omega \in \Omega, d((\widehat{f} X)(\omega),(\widehat{f} Y)(\omega))<q u\})>b_{n+1}
\end{gathered}
$$

where $\left(b_{n}\right)_{n \in \mathbb{N}}$ is a sequence from $(0,1)$ such that $\lim _{n \rightarrow \infty} b_{n}=1$ and $t$-norm $T$ defined by

$$
T(x, y)=\mathbf{s}^{-1}(\max (0, \mathbf{s}(x)+\mathbf{s}(y)-1)), x, y \in[0,1],
$$


satisfies condition

$$
\lim _{n \rightarrow \infty} \mathbf{T}_{i=n}^{\infty} b_{i}=1
$$

then there exists a random fixed point of the operator $f$.

\section{Authors' contributions}

T. Došenović has formulated Theorem 5 and Definition 10 which are the base for this paper. Also, together with A. Takači she has the Corollary's 1-4 of this theorem. Moreover, together with D. Rakić, M. Brdar Corollary 6 was formulated. The proofs are the joint contribution of all authors.

Finally, the authors declare that they have no competing interest considering the publication of this paper.

\section{Acknowledgements}

This research was supported by MNTRRS-174009 and Serbian Ministry of Education and Science project III 44006.

Received: 9 June 2011 Accepted: 31 October 2011 Published: 31 October 2011

\section{References}

1. Schweizer, B, Sklar, A: Probabilistic Metric Spaces. Elsevier North-Holland, New York (1983)

2. Čirić, LB, Mihet, D, Saadati, R: Monotone generalized contractions in partially ordered probabilistic metric spaces. Topol Appl. 156, 2838-2844 (2009). doi:10.1016/j.topol.2009.08.029

3. Mihet, D: Multivalued generalizatins of probabilistic contractions. J Math Anal Appl. 304, 464-472 (2005). doi:10.1016/j. jmaa.2004.09.034

4. Mihet, D: On the existence and the uniqueness of fixed points of Sehgal contractions. Fuzzy Sets Syst. 156, 135-141 (2005). doi:10.1016/j.fss.2005.05.024

5. Mihet, D: A note on a common fixed point theorem in probabilistic metric spaces. Acta Math Hungar. 125(1-2), 127-130 (2009). doi:10.1007/s10474-009-8238-3

6. Saadati, R, O'Regan, D, Vaezpour, SM, Kim, JK: Generalized distance and common fixed point theorems in Menger probabilistic metric spaces. Bull Iran Math Soc. 35(2), 97-117 (2009)

7. Žikić, T: Existence of fixed point in fuzzy structures. PhD thesis. University of Novi Sad, Faculty of Sciences and Mathematics (2002)

8. Hadžić, O, Pap, E: Fixed Point Theory in Probabilistic Metric Spaces, Theory in Probabilistic Metric Spaces. Kluwer, Dordrecht (2001)

9. Schweizer, B, Sklar, A: Statistical metric spaces. Pac J Math. 10, 313-334 (1960)

10. Hadžić, O: A fixed point theorem in Menger spaces. Publ Inst Math Beograd T. 20, 107-112 (1979)

11. Sehgal, VM, Baharucha-Reid, AT: Fixed points of contraction mappings on probabilistic metric spaces. Math Syst Theory. 6, 97-102 (1972). doi:10.1007/BF01706080

12. Mihet, D: Integalitatea triunghiului si puncte fixe in PM-spatii, Doctoral Thesis, West University of Timisoara, 1997, in English. (2001)

13. Klement, EP, Mesiar, R, Pap, E: Triangular Norms, Trends in Logic 8. Kluwer, Dordrecht (2000)

14. Hadžić, O, Pap, E, Budinčević, M: Countable extension of triangular norms and their applications to the fixed point theory in probabilistic metric spaces, uncertainty modelling. Kybernetika. 38(3), 363-382 (2002)

15. Mihet, D: A comparison of probabilistic contractions of Sehgal type. In: Seminar on Probability Theory and Applications (STPA) 145. West University of Timisoara http://www.math.uvt.ro/rom/pubs/preprints/stpa/stpa145.pdf (2003)

doi:10.1186/1687-1812-2011-74

Cite this article as: Došenović et al:: A fixed point theorem for a special class of probabilistic contraction. Fixed Point Theory and Applications 2011 2011:74. 